\title{
Efecto de la regulación de la cabecera del río Genil (Sierra Nevada, España) sobre la comunidad de macroinvertebrados acuáticos y la dieta larvaria de Rhyacophila nevada (Insecta: Trichoptera)
}

\author{
C. L. Bello ${ }^{1}$ y J. Alba-Tercedor ${ }^{2}$
}

1. Facultad de Ciencias. Departamento de Biología. Laboratorio de Limnología. Apdo. postal 10552. Santa Rita. Maracaibo. Venezuela.

2. Departamento de Biología Animal y Ecología. Facultad de Ciencias, Universidad de Granada. 18071Granada. España.

\begin{abstract}
RESUMEN
Se analiza el efecto que un embalse (Embalse de Canales), situado en la parte alta de un río de montaña (Río Genil, Sierra Nevada, Granada, España), tiene sobre la comunidad de macroinvertebrados, y en especial sobre la dieta de las larvas del tricóptero depredador Rhyacophila nevada Zamora-Muñoz, C. \& Alba-Tercedor, 1992. Para ello se establecieron estaciones de muestreo aguas arriba y abajo del embalse, realizándose muestreos cuantitativos de macroinvertebrados con una periodicidad mensual. Los macroinvertebrados (MIB) se identificaron hasta el máximo nivel taxonómico posible (en la mayoría de los casos a nivel de especie). La mayor diversidad específica se encontró en los dípteros quironómidos. Se estimó también la biomasa por especie (PSLC) y los ciclos de vida de las especies de depredadores, efemerópteros y dípteros. El contenido de los tractos digestivos fue analizado y cuantificado. La diversidad de los MIB estuvo determinada por los cambios de flujo y los ciclos de vida de las presas. Rhyacophila nevada es el depredador más abundante, presenta un ciclo multivoltino, comportándose como un depredador que busca activamente sus presas que ingiere comenzando por la región caudal. El número de presas en $R$. nevada aumentó con su tamaño. Las diferencias significativas de la dieta entre las estaciones de muestreo son consecuencia de los cambios en la disponibilidad de recursos (presas) en el tiempo. El ancho de nicho trófico también mostró diferencias entre estaciones.
\end{abstract}

Palabras claves: Insectos acuáticos, Trichoptera, ríos regulados, perturbación, depredación, alimentación, montaña.

\begin{abstract}
The effect that a dam (the Canales dam), located in the upper part of a mountain river (Genil stream, Sierra Nevada, Spain), has on the macroinvertebrate community, is analysed, especially over the larval diet of the predator caddisfly Rhyacophila nevada Zamora-Muñoz, C. \& Alba-Tercedor, 1992. To that end, sampling stations were established, upstream and downstream of the dam. Quantitative samplings of macroinvertebrates were realized every month. The macroinvertebrates were identified to the maximum taxonomical level (species level in the majority of cases). The highest specific diversity was found in the chironomidae Diptera. The biomass per species was estimated, as well as the life cycles of the predator's species, mayflies and dipterans. Gut contents were analysed and quantified. The diversity of the MIB was determined by flow shifts and prey life cycles. Rhyacophila nevada is the most abundant predator, with a multivoltine life cycle, and behaving as an active searcher of preys, ingesting them from the caudal region. The number of prey items consumed by R. nevada increased with its size. Significant differences on the diet between the sampling stations are a consequence of the shift in the resource availability of preys in time. The trophic niche breadth was different among sites.
\end{abstract}

Keywords: Aquatic insects, Trichoptera, regulated rivers, perturbation, predation, feeding, mountain.

\section{INTRODUCCIÓN}

Los factores físicos y químicos han sido señalados como los principales agentes extrínsecos de las comunidades bióticas de las aguas corrientes. Sin embargo en las últimas décadas el papel de las interacciones bióticas ha recibido gran atención. Existen dificultades para evaluar las 
tasas de renovación de las presas y la varianza que presentan las muestras, ya que el papel que juega la depredación en la trama trófica de los ríos puede presentar resultados contradictorios (Cooper et al., 1990).

Según Perckarsky \& Dodson (1980) la abundancia relativa de la presa o del recurso es la que define las interacciones entre un depredador y su presa. Por otro lado, diversos autores han señalado, que los depredadores cambian su dieta a lo largo de su ciclo de vida. En este sentido, Céréghino (2002) encontró cuatro especies del género Rhyacophila que cambian su dieta en función de su ciclo de vida. Resultados que coinciden parcialmente con lo encontrado en este trabajo para Rhyacophila nevada. Del mismo modo Bello (1991), en un río tropical intermitente, encontró un comportamiento similar para larvas de Corydalus sp. (Megaloptera) e Hydropsyche sp. (Trichoptera), lo que parece indicar que existe un comportamiento general de la mayoría de los depredadores en este sentido. La dieta varía con los cambios en la disponibilidad de los recursos en el tiempo y en el espacio, siendo por tanto el impacto de la depredación sobre la comunidad de presas, materia orgánica y algas, esporádico, y limitado a los grandes cambios sucesionales de la biota y sobre todo por efecto del flujo de los respectivos recursos (Bello, 1997).

Teniendo en cuenta los antecedentes previos, en el presente trabajo analizamos el efecto que la regulación de un río de montaña tiene sobre las comunidades de macroinvertebrados acuáticos, y en especial, sobre la abundancia y biomasa de las larvas del tricóptero depredador Rhyacophyla nevada.

\section{ZONA DE ESTUDIO}

El estudio se realizó en la parte alta del Río Genil (Sierra Nevada, Granada, España) en donde se sitúa el Embalse de Canales (terminado en 1988), situado a unos $10 \mathrm{kms}$ aguas arriba de la ciudad de Granada entre las poblaciones de Güejar Sierra y Pinos Genil. Presenta una

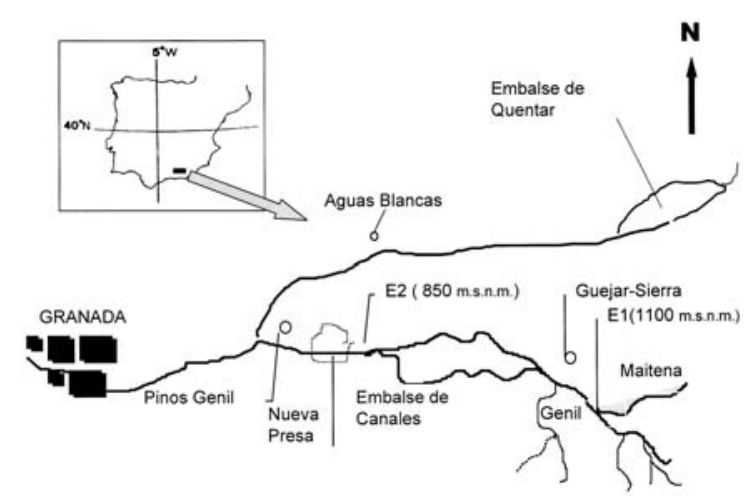

Figura 1. Área de estudio y situación de las estaciones de muestreo. Studied area and location of the sampling sites.

profundidad media de $38.2 \mathrm{~m}$. y sus valores de fósforo total y productividad primaria permiten caracterizarlo como un embalse eutrófico (Morales-Baquero et al., 1994).

Se situaron dos estaciones de muestreo (Fig. 1): una aguas arriba del embalse (E1, situada a 1100 $\mathrm{m}$. de altura sobre el nivel del mar y a unos $500 \mathrm{~m}$. aguas arriba del embalse) y otra aguas abajo del mismo (E2, situada a $850 \mathrm{~m}$. de altura y a unos $100 \mathrm{~m}$. aguas abajo de la presa del embalse).

\section{MATERIAL Y MÉTODOS}

En cada una de las estaciones de muestreo se tomaron muestras mensuales a lo largo de un ciclo anual comprendido entre el 27 de julio de 1992 y el 4 de julio de 1993. Los macroinvertebrados fueron colectados con red Surber, con una superficie de captura de $900 \mathrm{~cm}^{2}$, una profundidad de red de un metro y abertura de malla de $220 \mu \mathrm{m}$. Se tomaron seis muestras de Surber en cada estación. Los macroinvertebrados colectados junto con el sustrato, se colocaron en frascos, se les agregó Alka-Seltzer ${ }^{\circledR}$ para adormecerlos y evitar la regurgitación. Después de alrededor de cinco minutos se preservaron en formalina al $4 \%$ para su posterior separación y estudio en el laboratorio. Para el estudio del contenido digestivo, los ejemplares se colocaron en portaobjetos y se transparentaron mediante el método de Bello \& Cabrera (1999). Para la cuantificación del conte- 
nido del tracto digestivo se empleó el método de los puntos (Lavandier, 1979), método que según Hansson (1970) resulta significativo para grandes muestras. Los insectos que no fueron montados en portaobjetos se emplearon para determinar el peso seco libre de cenizas (PSLC) por métodos gravimétricos. Para ello se realizaron pesadas por separado de los diferentes estadíos de desarrollo. Así, Rhyacophila nevada (Zamora-Muñoz \& Alba-Tercedor, 1992) se pesó por clases de tamaño de acuerdo a su ancho cefálico. Las otras especies se pesaron de acuerdo a su tamaño, los gran- des de manera individual o en grupos de $5,10 \mathrm{o}$ más cuando eran muy pequeños, los efemerópteros de acuerdo a sus fases de desarrollo (AlbaTercedor, 1990 a, b). Se utilizaron algunos ejemplares de $R$. nevada para establecer la relación tamaño-peso. El peso de los componentes de la dieta de $R$. nevada se realizó por el método de los pesos reconstruidos (Bagenal, 1978) y se utilizó para el cálculo del ancho de nicho trófico el índice de Shannon-Weaver (1949), aplicado a los componentes de la dieta de acuerdo al peso seco libre de cenizas de cada presa.
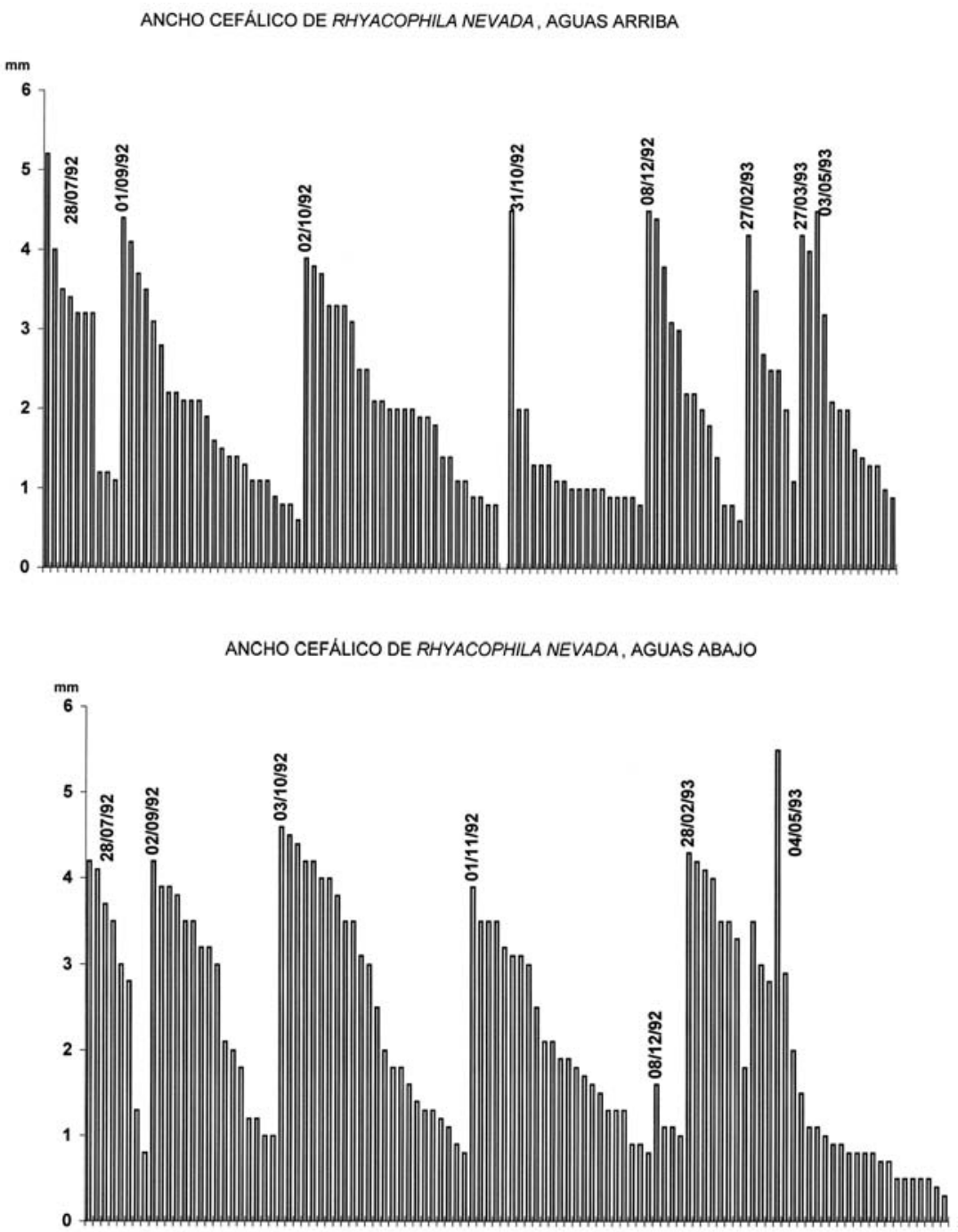

Figura 2. Distribución mensual del ancho cefálico de R. nevada, en cada una de las estaciones de muestreo. Monthly distribution of the R. nevada cephalic width at each sampling site. 


\section{RESULTADOS}

El análisis del contenido de los tractos digestivos permitió clasificar a seis especies como depredadoras aguas arriba (el tricóptero: $R$. nevada, el plecóptero: P. marginata y cuatro especies de dípteros: Conchapelopia pallidula, Conchapelopia sp. 2, Limnophora sp. y Procladius sp.) y a cuatro especies depredadoras aguas abajo (el tricóptero: $R$. nevada, y tres especies de dípteros: Conchapelopia pallidula, Limnophora sp. y Procladius sp.). R. nevada fue el depredador más abundante, representando el $77 \%$ y $90 \%$ de los individuos depredadores existentes en las estaciones de aguas arriba y aguas abajo del embalse respectivamente. $R$. nevada es una especie con
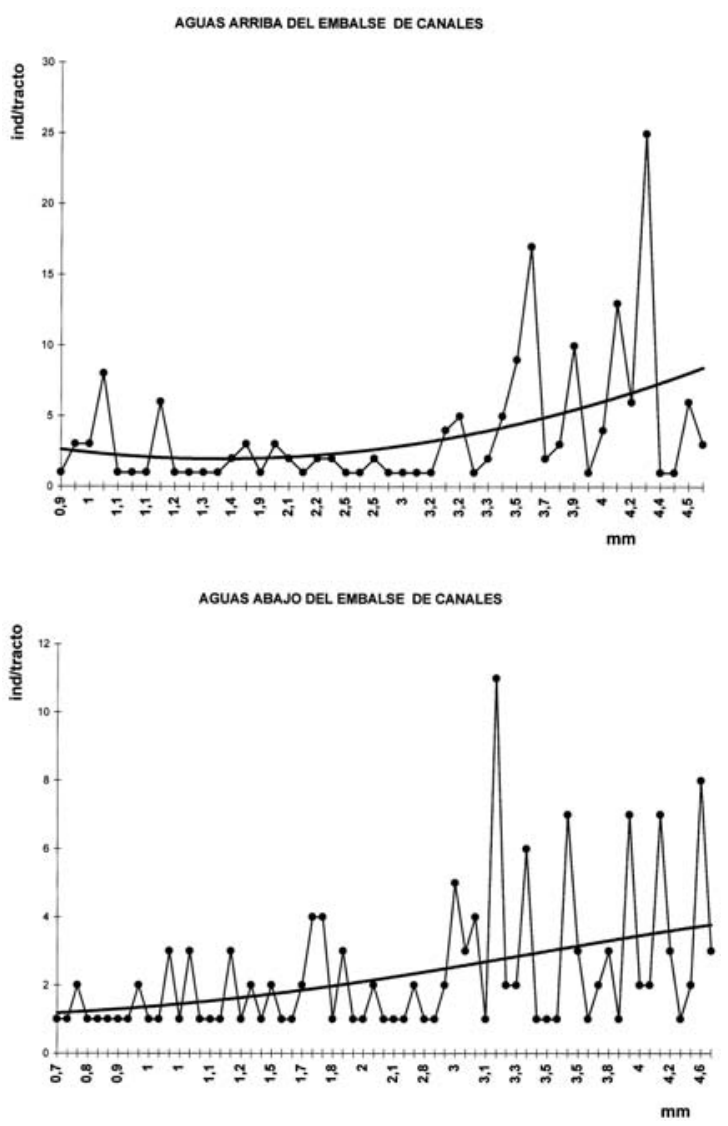

Figura 3. Número de presas en el tracto digestivo de $R$. neva$d a$ de acuerdo al ancho cefálico en las estaciones de muestreo. Number of prey in the digestive tract of $\mathrm{R}$. nevada, according to the cephalic width at each sampling site.
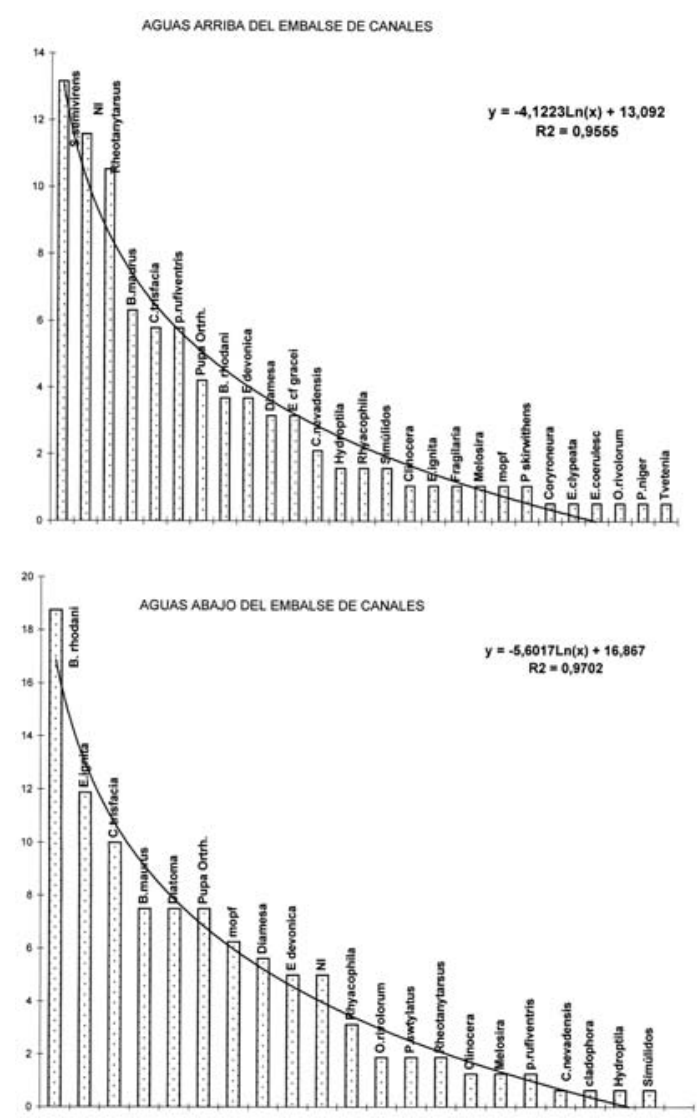

Figura 4. Frecuencia relativa de las presas en el contenido del tracto digestivo de $R$. nevada en las estaciones de muestreo. Relative frequency of prey in the digestive tract of $\mathrm{R}$. nevada at each sampling site.

crecimiento continuo (Fig. 2). Una prueba $t$ no arrojó diferencias significativas de los anchos cefálicos de Rhyacophila nevada entre las dos estaciones $(\mathrm{p}=0.0001)$.

Según nuestras observaciones. $R$. nevada engulle las presas comenzando por la parte caudal. Otras observaciones experimentales encontraron un comportamiento similar en los Limnephilidae (Trichoptera) (Giller \& Sangpradub, 1993). $R$. nevada tritura las presas lo que dificulta la medida del ancho cefálico de las mismas. Este efecto de trituración puede dar origen a errores en la interpretación de la dieta de los depredadores debido al alto contenido de algas, principalmente diatomeas, en la mayoría de los 


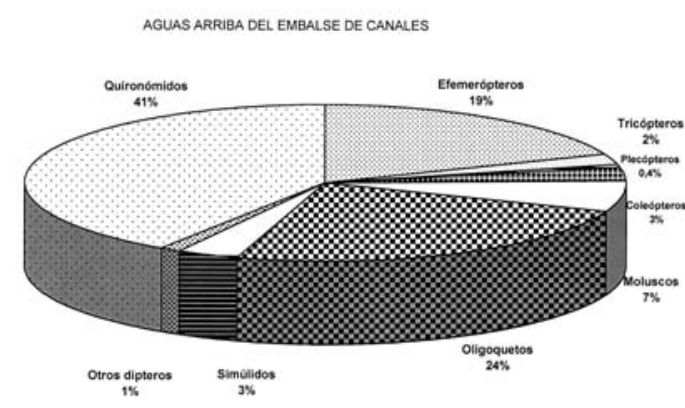

AgUAS ABANO DEL EMBALSE DE CANALES

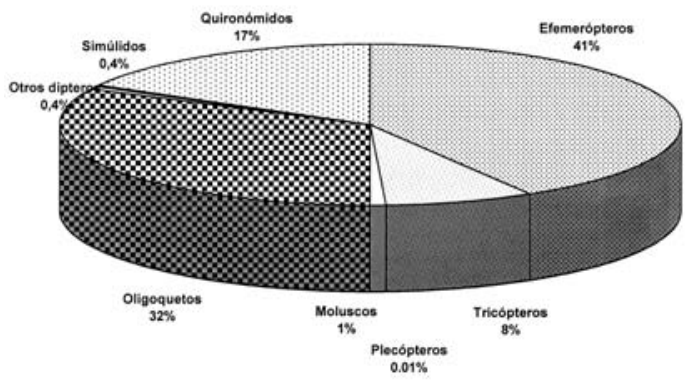

Figura 5. Comparación porcentual anual de los taxones en las estaciones de muestreo. Annual percentage comparison of the taxa in the sampling sites.

quironómidos y otros invertebrados presa. El número de presas capturadas aumentó con el tamaño de $R$. nevada (Fig. 3). Según Dudgeon \& Richardson (1988) $R$. inculta no consume más de una presa cada vez y en sus tractos no observaron individuos de más de dos taxones. Por el contrarion, Céréghino (2002) encontró de dos a cinco presas en los estadios III y IV y pertenecientes a dos taxones. Nosotros encontramos, aguas arriba del embalse, un ejemplar de ancho cefálico de $4.4 \mathrm{~mm}$, con 25 presas de diferentes taxones; tricópteros, efemerópteros y dípteros. Asimismo encontramos diferencias en la dieta entre las estaciones de muestreo (Fig. 4). Aguas arriba los quironómidos fueron dominantes en los tractos $(74 \%)$, mientras que aguas abajo los efemerópteros (39\%) y los quironómidos (35\%) fueron los grupos dominantes. Estos cambios en la dieta deben estar relacionados con los cambios en las abundancias relativas de esos
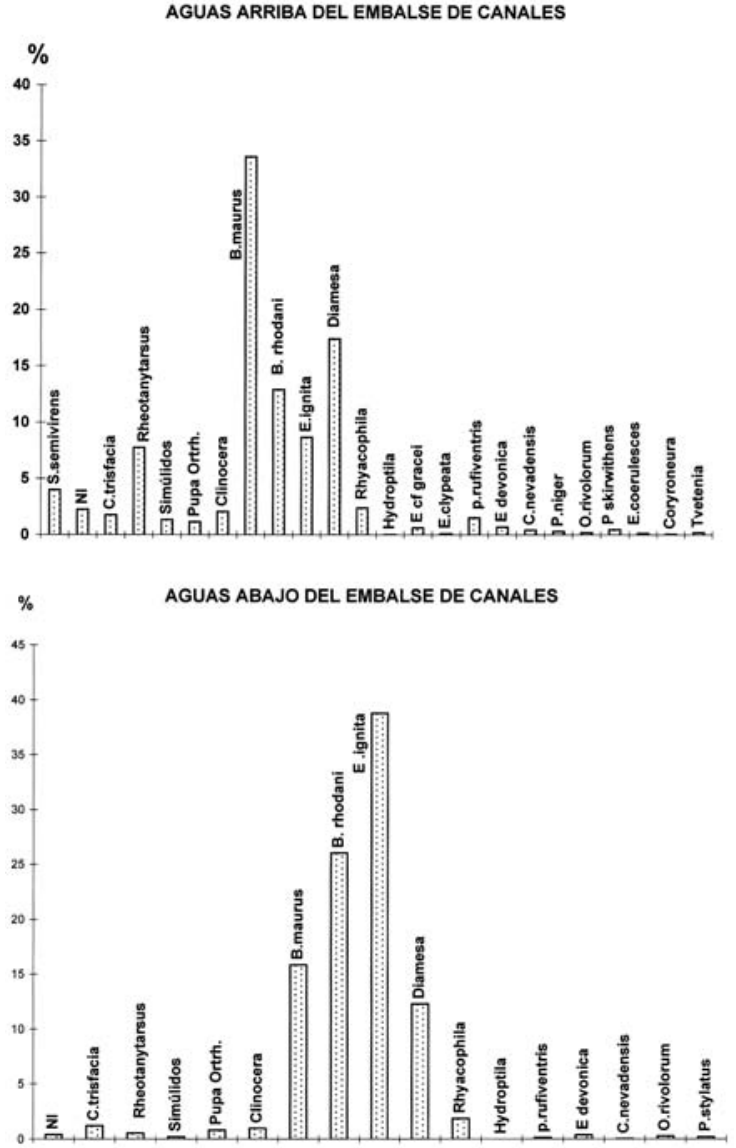

Figura 6. Biomasa relativa de los macroinvertebrados que forman parte de la dieta de $R$. nevada en las estaciones de muestreo. Relative biomass of macroinvertebrate in the diet of $\mathrm{R}$. nevada at the sampling sites.

grupos (Fig. 5). Las diferencias observadas de la biomasa de las presas refleja la importancia de los efemerópteros en ambas estaciones (Fig. 6) aunque sin olvidar que los exoesqueletos que contribuyen de manera apreciable en la biomasa son poco utilizados energéticamente por los depredadores. Por ejemplo aguas abajo la especie más importante en los tractos por su biomasa fue Ephemerella ignita (Ephemeroptera). Las especies consumidas por $R$. nevada aguas arriba fueron distintas a las consumidas aguas abajo del embalse (Fig. 6). Aguas arriba la especie más depredada fue Sinorthocladius semivirens (Orthocladino, Díptero). Aguas abajo 
la especie más depredada fue Baetis rhodani (Ephemeroptera).

La abundancia de las presas en cada red de Surber y en cada estación, se relaciona positiva o negativamente con la abundancia de esa especie en el tracto de la especie depredadora (Figs. 7, 8). Por ejemplo, $S$. semivirens presentó sus máximos de abundancia en la estación aguas arriba en los muestreos del 27/7/92 y 1/9/92 que coincidió con los meses en los cuales fue la especie más depredada. De igual forma ocurrió con $B$. rhodani. Por el contrario E. ignita no fue depredada cuando fue más abundante. Lo inverso sucedió con el efemeróptero Baetis maurus, poco abundante aguas arriba, pero que resultó ser una de las especies más depredadas. Asimismo, se observó un comportamiento de canibalismo por parte de $R$. nevada, en ambas estaciones de muestreo, posiblemente propiciado por la presencia simultánea de distintas clases de tamaño (edad).

En vista de estos resultados se realizó un análisis de regresión lineal simple entre cada especie y el Tricóptero, buscando el mejor modelo que explicará la relación entre estas especies presa y el predador. Los resultados obtenidos aguas arriba del embalse fueron los siguientes: B. rhodani, $\mathrm{r}=0.32 ; \mathrm{r}^{2}=3.95, \mathrm{p}=0.1151 ; \mathrm{n}=$ 63; no significativo (N.S.), E. ignita, $\mathrm{r}=0.863$; $\mathrm{r}^{2}=75.047, \mathrm{p}=0.0000 ; \mathrm{n}=45$; significativo $(\mathrm{S})$; varianza $\left(\mathrm{s}^{2}\right)=1$ 739. Aguas abajo, B. rhodani, $\mathrm{r}=0.40 ; \mathrm{r}^{2}=8.41, \mathrm{p}=0.001 ; \mathrm{n}=62 ;(\mathrm{S})$; $\mathrm{s}^{2}=86108$, B. maurus, $\mathrm{r}=0.47 ; \mathrm{r}^{2}=22.66$, $\mathrm{p}=0.0003 ; \mathrm{n}=53 ;(\mathrm{S}) ; \mathrm{s}^{2}=3355$, efemerópteros totales, $\mathrm{r}=0.41 \mathrm{r}^{2}=16.82, \mathrm{p}=0.0007 ; \mathrm{n}=64$; $(\mathrm{S}) ; \mathrm{s}^{2}=99$ 284. Aguas arriba "n" total presas = 23 741; "n" de R. nevada = 301; Aguas abajo, "n" presas $=30$ 465; "n" de $R$. nevada $=496$.

Las otras especies consumidas por R. Nevada presentaron variaciones importantes en la varianza.

La comparación del ancho de nicho trófico de $R$. nevada, medido en base al peso seco libre de cenizas de las presas (Magurran, 1988), aguas arriba $\left(\mathrm{H}^{\prime}=2.149, \mathrm{~J}^{\prime}=0.67\right)$ y aguas abajo $\left(\mathrm{H}^{\prime}=\right.$ $1.6, \mathrm{~J}$ ' $=0.57)$ evidencia que aguas arriba la discriminación entre presas fue menor, probablemente por la presencia de un mayor número de especies pioneras (quironómidos).

\section{DISCUSIÓN}

Aguas arriba del embalse, durante el periodo de estudio hubo seis crecidas que determinaron en gran medida la alternancia de las presas encontradas en las redes de Surber y en los tractos digestivos de $R$. nevada. Aguas abajo, la presencia del alga Cladophora glomerata y sus distintas fases de desarrollo, así como la presencia de una comunidad de diatomeas asociada a la macroalga que define a esta comunidad como "Cladophora Glomeratum" (Margaleff, 1983), determina en gran medida la alternancia de especies. Hay que destacar que los máximos de abundancias medias o altas de las especies de quironómidos no coincidieron temporalmente en ambas estaciones de muestreo. Esto sugiere alteraciones de los mecanismos de repoblación por adultos a pesar de la poca distancia entre ambas estaciones. Aguas abajo $B$. rhodani ofrece una fuente segura de alimento para el predador $R$. nevada; ya que presenta un ciclo de vida de tipo multivoltino, por lo que hubo disponibilidad de ninfas pequeñas (clases de tamaño 1 y 2) en todos los meses de muestreo (Bello, 1997).

No se encontraron evidencias claras de que en áreas de mayor densidad de presas ocurra una mayor depredación (en las redes de Surber), lo que coincide con observaciones de otros autores (Hildrew \& Towsend, 1982; Sih, 1987; Peckarsky \& Penton, 1989; Jonhansson,1993). Los resultados de estos autores sugieren una relación positiva y que los depredadores no afectan la densidad de las presas. Nuestros resultados mostraron escasas relaciones y correlaciones positivas entre el depredador y las presas ya que de veintitres (23) especies consumidas, sólo una especie mostró asociación aguas arriba, y de dieciséis (16) especies consumidas aguas abajo solo dos especies presentaron asociación. Aguas arriba del embalse la especie asociada (E. ignita) no fue la más depredada (Fig. 5) pues $R$. nevada consumió quironómidos en mayor proporción (79.2\%), debido probablemente a sus ciclos de vida corto y a que cada especie incrementa 

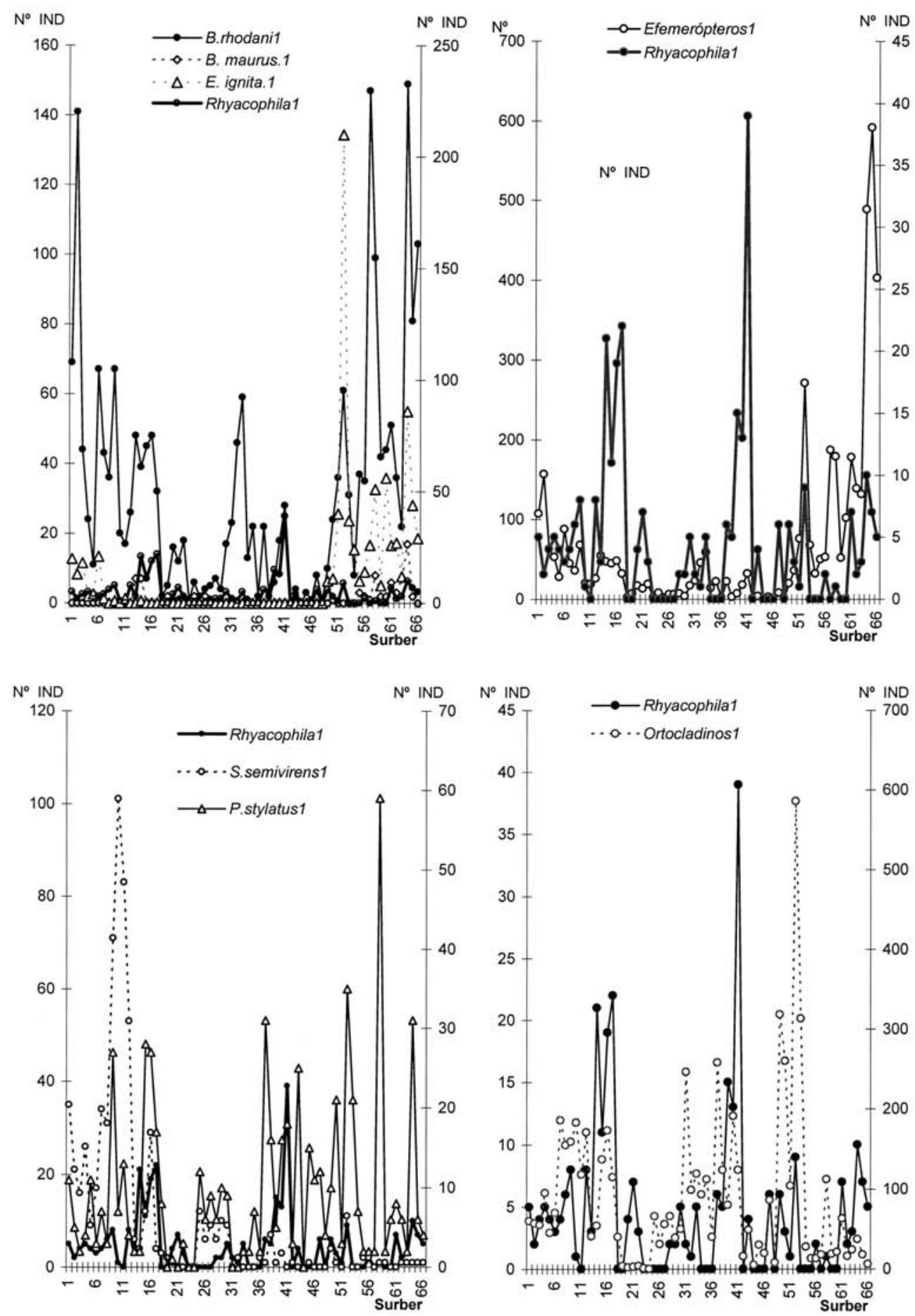

Figura 7. Abundancias de las presas y de $R$. nevada (efemerópteros y dípteros quironómidos) en cada muestra de Surber en E1. Cada seis muestras representan un mes, consecutivamente desde Julio/1992 hasta Junio/1993. Prey and R. nevada (mayflies and chironomids) abundances in each Surber sample on E1. Every six samples represent a month, consecutively since July/1992 to June/1993. 


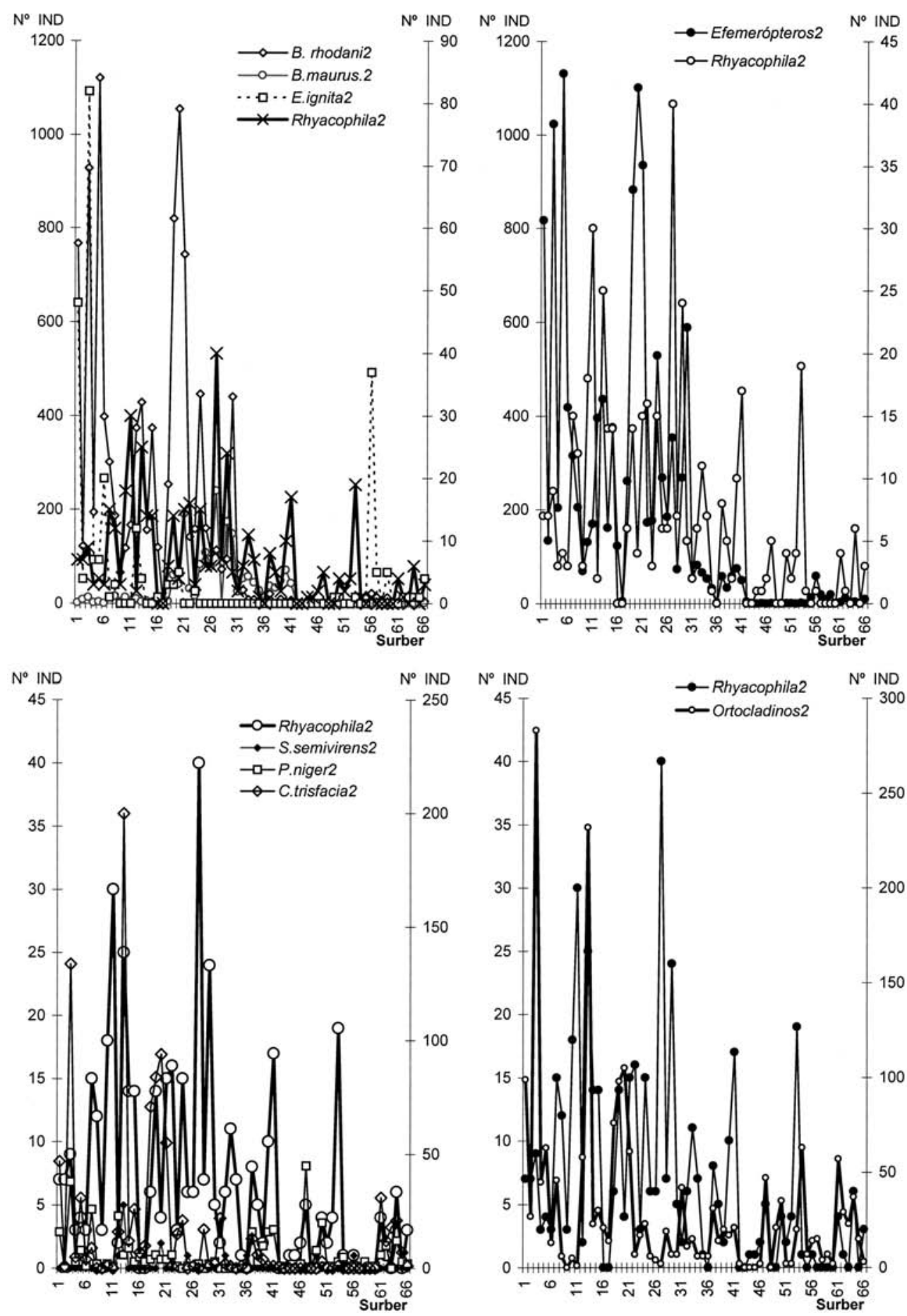

Figura 8. Abundancias de las presas y de $R$. nevada (efemerópteros y dípteros quironómidos) en cada muestra de Surber en E2. Cada seis muestras representan un mes, consecutivamente a partir de Julio/1992 hasta Junio/1993. Preys and R. nevada (mayflies and chironomids) abundance in each Surber sample on E2. Every six samples represent a month, consecutively since July/1992 to June/1993. 
transitoriamente sus abundancias (pioneras) con las perturbaciones del flujo (Bello, 1997).

Se debe tener en cuenta a su vez, que los altos valores de varianza observados en los efemerópteros y en general en la comunidad de macroinvertebrados consumidos impiden reflejar el efecto de la depredación sobre la comunidad de presas. Las pocas correlaciones encontradas pueden ser consecuencia de la baja abundancia relativa $(1.2 \%$ a $1.6 \%)$, el ciclo de vida y la estrategia de alimentación del depredador, en comparación con la abundancia relativa $(98.2 \%$ a $98.8 \%$ ), la tasa de renovación y las alternancias de las presas. La tasa de consumo del depredador es menor que la tasa de renovación de las presas.

Los resultados obtenidos por Peckarsky et al., (1994) sugiriendo que Ephemerella ignita es poco depredada debido a tener cuerpos duros con espinas, no se corrobora con nuestros resultados frente al tricóptero depredador $R$. nevada aguas abajo del embalse, lo que indica que no depende de los exoesqueletos y su valor energético, sino de una mayor probabilidad de captura posiblemente de los estadios primarios de $E$. ignita que no se diferencian apreciablemente de un Baetis. Se debe tener en cuenta, que además del animal en sí, el contenido de su tracto digestivo, puede jugar un papel importante en la energética alimentaria de los depredadores. En los tractos observados, y en particular en los quironómidos, la abundancia de diatomeas era muy alta lo que aporta al depredador una energía de excelente calidad.

El caudal aguas arriba presentó fuertes variaciones (35 días con más de dos veces el caudal promedio anual). Aguas abajo el flujo fue más constante (ningún día con más de dos veces el promedio del flujo anual). Igual ocurrió con el sustrato de rocas, arenas y la presencia de la macroalga Cladophora glomerata. Si se considera que aguas abajo el ambiente fue relativamente más estable, las interacciones bióticas deberían prevalecer (regresiones negativas), lo cual no fue evidente en nuestros resultados como se explico anteriormente. Sugerimos que el dominio en abundancia de los efemerópteros podría ser el resultado del ambiente más estable aguas abajo.
Las regresiones positivas o la ausencia de las mismas entre $R$. nevada y sus presas parecen indicar que no hay evidencias de la disminución de los recursos (predador-presa) debido probablemente a la alta tasa de renovación y alternancia de los quironómidos, al ciclo de vida continuo de los efemerópteros, la movilidad y abundancia del depredador, la varianza de las muestras y al caudal de la corriente. Esto sugiere, como poco probable y verificable, un mecanismo de regulación tipo "top-down" en las condiciones naturales de los ecosistemas lóticos.

\section{REFERENCIAS}

ALBA-TERCEDOR, J. 1990a. Life cycle and ecology of mayflies from Sierra Nevada (Spain), IV. Limnetica, 6:23-34.

ALBA-TERCEDOR, J. 1990b. Life cycles and ecology of some species of Ephemeroptera from Spain. In: Mayflies and Stoneflies: Life Histories and Biology. I.C. Campbell, (ed.):13-16., Serie Entomologica, 44. Kluwer Academic Publishers. Dordrecht.

BAGENAL, T. 1978. Methods for assessment of fish production in fresh water. IBP, Handbook 3. Oxford. Blackwell Scientific: 101-136.

BELLO, C.L. 1991. Análisis de la comunidad de insectos acuáticos del Caño Paso del Diablo (Guasare, Edo. Zulia) con especial referencia a los Ephemeroptera y Trichoptera. Trabajo de Ascenso. Universidad del Zulia.

BELLO, C. L.1997. Alteraciones de procesos ecológicos en un río de montaña como consecuencia de su regulación. Incidencia sobre los macroinvertebrados bénticos. Tesis Doctoral. Universidad de Granada. España. 286 pp

BELLO, C. L. \& M. I. CABRERA. 1999. Uso de la técnica microhistológica de Cavender y Hansen en la identificación de insectos acuáticos. Bol. Entomol. Venez., 14(1): 77-79.

CÉRÉGHINO, R. 2002. Shift from a herbivorous to a carnivorous diet during the larval development of some Rhyacophila species (Trichoptera), Aquatics insects, 24(2): 129-135.

COOPER, S. D., S. J. WALDE \& B. L. PECKARSKY. 1990. Prey exchange rates and the impact of predators on prey populations in streams. Ecology, 71(14): 1053-1514. 
DUDGEON, D. \& J. S. RIHARDSON. 1988. Dietary variations of predaceous caddisfly larvae (Tricoptera:Rhyacophilidae, Polycentropodidae and Arctopsychidae) from British Columbian streams. Hydrobiologia, 160: 33-43.

GILLER, P. S. \& N. SANGPRADUB. 1993. Predatory foraging behavior and activity patterns of larvae of two species of limnephilid cased caddis. Oikos, 67: 351-357.

HANSSON, L. 1970. Methods of morphological diet microanalysis in rodents. Oikos, 21: 225-266.

HILDREW, A. G. \& C. R. TOWNSEND. 1982. The distribution of two predators and their prey in an iron rich stream. J. Anim. Ecol., 45: 41-57.

JOHANSSON, F. 1993. Effects of prey type, prey density and predator presence on behaviour and predation risk in a larval damselfly. Oikos, 68(3): 481-489.

LAVANDIER, P. 1979. Ecologie d'un Torrent Pyreneen de Haute Montagne: L'Estaragne. Tesis Doctoral. L'Université Paul Sabatier de Toulouse. 532 pp

MAGURRAN, A. E. 1988. Ecological Diversity and Its Measurement. Princeton University Press. 179 pp.

MARGALEF, R. 1983. Limnología. Ed.Omega. Barcelona. España. 1010 pp.

MORALES-BAQUERO, R., J. M. CONDEPORCUNA \& L. CRUZ-PIZARRO. 1994. The zooplancton biomass and food availability in four reservoirs of contrasting trophic status. Arch. Hydrobiol. Beih., 40: 161-173.

PECKARSKY, B. L., C. A. COWAN \& C. R. ANDERSON. 1994. Consequences of the specialized predatory behavior of stream-dwelling stonefly larvae. Ecology, 75(1): 166-181.

PECKARSKY, B. L. \& S. DODSON. 1980. Do stonefly predator influence benthic distributions in streams? Ecology, 61(6): 1275-1282.

PECKARSKY, B. L. \& M. A. PENTON. 1989. Mechanisms of prey selection by stream-dwelling stoneflies. Ecology, 70(5): 1203-1218.

SHANNON, C. E. \& W. WEAVER. 1949. The mathematical theory of communication. The Univ. of Illinois Press. Urbana, IL. 107 pp.

SIH, A. 1987. Predators and prey lifestyles: An evolutionary and ecological overview. In: Predation: Direct and Indirect Impacts on Aquatic Communities. Kerfoot, W.C. \& Sih, A. (eds.): 203224. University Press, New England.

ZAMORA-MUÑOZ, C. \& J. ALBA-TERCEDOR. 1992. Description of the Larva of Rhyacophila (Rhyacophila) nevada Schmid, 1952 and key to the species of Rhyacophila of the Iberian Peninsula (Trichoptera:Rhyacophilidae). Aquatic Insects, 14(2): 65-71. 